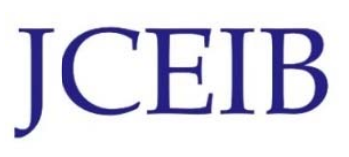

Journal Chemical Engineering and Industrial Biotechnology (JCEIB)

Open Access

Volume 4 pp. 10-18; September 2018

(C)Universiti Malaysia Pahang Publisher

DOI: https://doi.org/10.15282/JCEIB-V4-11.30/9/2018.4.4

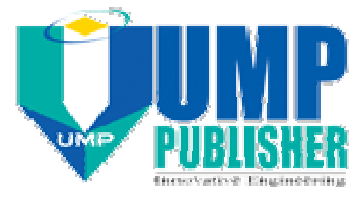

\title{
OPTIMIZATION OF BETA-SITOSTEROL EXTRACTION FROM LEUCAENALEUCOCHEPALA BY MICROWAVE ASSISTED HYDRODISTILLATION
}

\author{
Norlaili Hashim’ Noormazlinah Ahmad,*A.M. Mimi Sakinah, A. Maria-Pilar \\ Faculty of Chemical and Natural Resources Engineering, Universiti Malaysia Pahang, Lebihraya Tun \\ Razak, 26300 Gambang,Kuantan, Pahang, Malaysia \\ Faculty of Engineering Technology, Universiti Malaysia Pahang, Pahang, Malaysia \\ Department of Chemical Engineering (EQ), Universitat Polytechnicia of Catalunya, Barcelona, Spain \\ * Corresponding author: E-mail: mazlinah@ump.edu.my \\ Tel.: +609-5492868
}

\begin{abstract}
Microwave-assisted hydrodistillation (MAH) extraction process can extract phytosterol(beta-sitosterol) from Leucaena leucochepala. The suitable parameter of the extraction process to extract the highest yield of phytosterol from a plant can be optimized by using central composite design (CCD). Two parameter extraction process were studied which are duration of time (4 to 8 minutes) and solvent ethanol concentration (55\% to 95\%) to gain the highest yield of extracted beta-sitosterol from Leucaena leucochepala. The Liebermann-Burchard analysis was used to analyse phytosterol content in the extracted from the samples. The CCD model parameter was significant because of the values for Prob $>\mathrm{F}$ is less than 0.0500 which are 0.006 and value lack of fit F-value which is 5.44 represent only $6.77 \%$ chances to occur failure. This indicated the CCD with duration of extraction and the ethanol solvent concentration is the optimum parameter for the extraction of beta-sitosterol(phytosterol) from Leucaena leucochepala legume pod with the highest yield of phytosterol of $0.2717 \mathrm{mg} / \mathrm{mL}$ at $75 \%$ ethanol and at 6 minutes.
\end{abstract}

Keywords: phytosterol; microwave assisted hydrodistillation extraction; central composite design; Liebermann Burchard; Leucaena leucochephala.

\subsection{INTRODUCTION}

Leucaena Leucocephala is the scientific name for Petai Belalang. It is available all year long and can be consumed as a salad in Malay culture. It can reproduce in a large volume in a short period of time. According to International Union for Conservation of Nature and Natural Resources (IUCN), L. leucocephala listed in 100 aggressive species where this type of plant can cause severe harm to the biological environment ( $\mathrm{Li}$ et al., 2012). Thus, it such a waste for not fully utilized an abundant source of raw material. It has come with some valuable material to human health such as a natural source of antioxidant and cytotoxicity (Aderogba et al., 2010), has an antidiabetic material (Syamsudin et al., 2010), and come with phytosteroid (Chen \& Wang, 2010). 
The extraction process can be done by another process such as maceration, Soxhlet extraction and microwave-assisted hydrodistillation. MAH is a type of process can give a better end result yield but with lower energy usage (Farhata et al., 2009). Microwave heating method also provide a greater heating process because of the heating process not only occur at the surface of the material but also occur at entirely and depth of the material (Wang \& Weller, 2006). MAH process also can contribute in order to reach a greener technology as their chemical reaction are gaining an important role in the organic synthesis (Begum et al., 2015).

In the extraction process, each parameter can be depended to each other in the tested design.in order to reach the optimum level of the desired result, information on the relationship between each parameter is very important. As for this reason, one-factor-ata-time method become is not inappropriate because this type of method does not provide the interaction between each parameter (Elksibi et al., 2014). But, response surface method (RSM) such as Central Composite Design (CCD) can give the quantitative data that can provide estimation on the connection between several parameters from the model equation generated from the design model. The optimization process of the design model, central composite design (CCD), can provide the better result as compared to another old-fashioned bioprocess optimization method (Palukurty \& Somalanka, 2016).

The most significant parameter of MAH for extracting of phytosterol(beta-sitosterol) from L. Leucocephala is ethanol solvent concentration and duration of extraction. CCD is an empirical model that acts as a tool to optimise the most significant parameter to obtain the highest yield.

\subsection{METHODS AND MATERIALS}

\subsection{Plant sample preparation}

L. leucocephala legume pod was collected from Pekan and Kuantan in the year of 2016 because this plant can grow wild and rapidly. Then, by using distilled water, the legume pod was prewashed thoroughly and dried using the paper towel. by using the sample grinder, the legume pod was ground to increase the surface area to contact with solvent extraction (Namiesnik et al., 2003).

\subsection{Extraction process}

Microwave-assisted hydrodistillation(MAH) system was used to perform the extraction process. For each extraction process, 20g of pulverized plant sample was weighed to run the extraction (Ren et al., 2015). Weighed sample was transferred then into microwave $1000 \mathrm{~mL}$ round bottom flasks. Later, $200 \mathrm{~mL}$ solvent extraction was added into the flask. The MAH extraction system was equipped with a temperature control device that can adjust the system temperature and condensing unit for the solvent solution (Ren et al., 2015). The cooling system was set at $16^{\circ} \mathrm{C}$ to gain the maximum effect of solvent condensation. experiment run was performed for 23 CCD run where 8 runs of the factorial point, 6 axial points and 5 repetitions of the centre point in a single block. This process was performed to optimizes the most significant parameter to obtain the maximum yield of phytosteroid. Table 3.4 shows the two significant independent variables and their level for the CCD experimental design. For each extraction run, the extraction condition was set to the desired extraction condition for the duration of 
extraction and at the different solvent concentration in Table 1 . The power of the extraction process was set at $600 \mathrm{~W}$ and temperature at $75^{\circ} \mathrm{C}$.

Table 1: Extraction condition for the CCD process

\begin{tabular}{llcc}
\hline Parameters & Unit & -1 & Level \\
\cline { 3 - 4 } & & 55 & +1 \\
\hline$\%\left(\mathrm{x}_{1}\right)$ solvent concentration & $\mathrm{V}: \mathrm{V}$ & 4 & 95 \\
$\mathrm{~B}\left(\mathrm{x}_{2}\right)$ time of extraction & minute & 4 & 8 \\
\hline
\end{tabular}

2.4 Liebermann-Burchard reagent

$5 \mathrm{~mL}$ of acetic anhydride was transferred into an amber vial and left in an ice bath for 30 minutes. After that, $0.5 \mathrm{~mL}$ of sulphuric acid was added carefully into the ice-cold acetic anhydride.

2.5 Sample quantitative analysis

$0.5 \mathrm{~mL}$ chloroform and $4 \mathrm{mLethanolic}$ water solvent were added into each cuvette. Then, $0.1 \mathrm{~mL}$ of reduced volume sample extraction was added. After that, 2 drops of Liebermann-Burchard reagent was added to the solution. Chloroform was added to adjust the solution volume. The absorption of each sample was measured by using UV/Vis after 5 min addition of LB reagent. Chloroform was used as the measured blank. The quantitative method to measure the phytosterol content in the extracted sample was also supported by another study by L. B. D. C. Araujo et al., 2013. The UVVis analysis was used to measure the phytosterol concentration in the plant extracted sample at $625 \mathrm{~nm}$.

2.6 Scanning Electron Microscope (SEM) analysis

JSM 7800F FESEM facility was used to perform the imaging analysis of the $L$. leucochepala legume pod before and after the extraction process. The sample was captured for some magnification level at 500X, 1000X and 2000X. The system used for the analysis is EDX X-MAXN OXFORD system and was done on electron microscope JEOL, JSM 7800F USA that comes with a thermal field emission SEM high purity germanium energy dispersive X-ray detector for capturing and observing the structure surface of the sample (Kuppusamy et al., 2014).

\subsection{RESULTS AND DISCUSSIONS}

The colour changes in the tested sample after the addition of chloroform sulphuric acid and acetic anhydride from violet blue to green colour indicate the presence of phytosterol in the plant sample (Harborne, 1998). The analysis was set at the wavelength value of $625 \mathrm{~nm}$ because maximum blue oxidation process occurs at $625 \mathrm{~nm}$ (Araujo et al., 2013). The result of the beta-sitosterol concentration present in the plant sample was in Table 2. 


\begin{tabular}{lllll}
\hline Standard & Run & Concentration $(\mathrm{V}: \mathrm{V})$ & Time (min) & $\begin{array}{l}\text { Phytosteroid } \\
\text { concentration }(\mathrm{mg} / \mathrm{mL})\end{array}$ \\
\hline 12 & 1 & 75.00 & 6.00 & 0.2609 \\
$\mathbf{3}$ & $\mathbf{2}$ & $\mathbf{7 5 . 0 0}$ & $\mathbf{6 . 0 0}$ & $\mathbf{0 . 2 7 1 5}$ \\
13 & 3 & 75.00 & 3.17 & 0.2656 \\
1 & 4 & 75.00 & 6.00 & 0.2421 \\
9 & 5 & 75.00 & 6.00 & 0.2678 \\
7 & 6 & 75.00 & 8.83 & 0.2652 \\
2 & 7 & 75.00 & 6.00 & 0.2089 \\
11 & 8 & 55.00 & 4.00 & 0.2224 \\
6 & 9 & 55.00 & 8.00 & 0.245 \\
4 & 10 & 95.00 & 4.00 & 0.2507 \\
8 & 11 & 95.00 & 8.00 & 0.0132 \\
10 & 12 & 46.72 & 6.00 & 0.255 \\
\hline 5 & 13 & 100.00 & 6.00 & 0.0125 \\
\hline
\end{tabular}

Table 2 : CCD Experimental results beta-sitosterol extraction

Figure 1.1 and 1.2 shows the surface of L. leucocephala legume pod before and extraction process. It was shown in the after extraction process surface image, the structure was crimpled and broken down. Whereas, the image for the raw sample surface shows the existent of the cellular wall that likely similar to the image of cellulose and hemicellulose structure (Gomez et al., 2008). This proven extraction process can break down the sample structure and eventually permitting the desired product to be extracted. Chi et al., 2011, also verified that by breaking down the plant sample structure MAE also succeeded in extracting the desired product from plant sample.

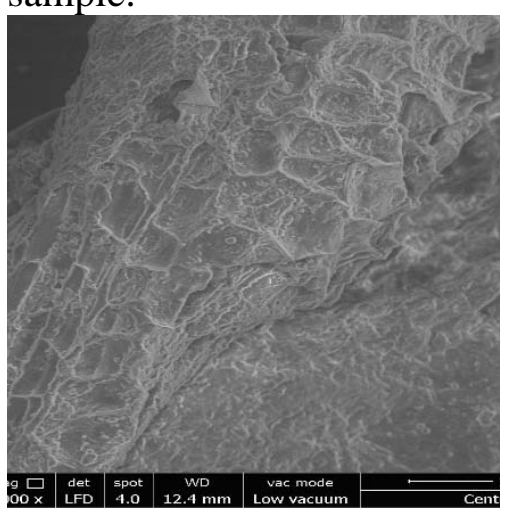

Figure 1.1: L. leucocephala surface structure before extraction process

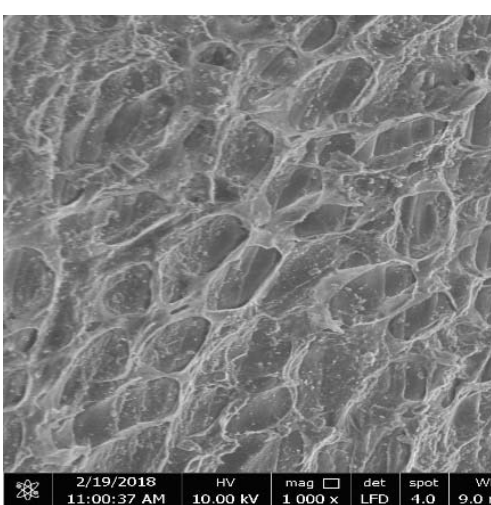

a) L. leucocephala

Figure2 shows the calibration curve for the phytosterol concentration in Leucaena leucocephala at different concentration ranges from 0.02 to $0.10 \mathrm{mg} / \mathrm{mL}$ and generate Eqn. 1. 


$$
y=m x+c
$$

$y=$ absorbance value, $\mathrm{m}=$ slope of graph (6.3117), $\mathrm{c}=$ constant value (0.0124)

Another research has been done based on this calibration curve to study the phytosterol concentration in the roots of Acanthopsermumhispidium by using UV-Vis spectrophotometry (L. B. D. C. Araujo etal., 2013).

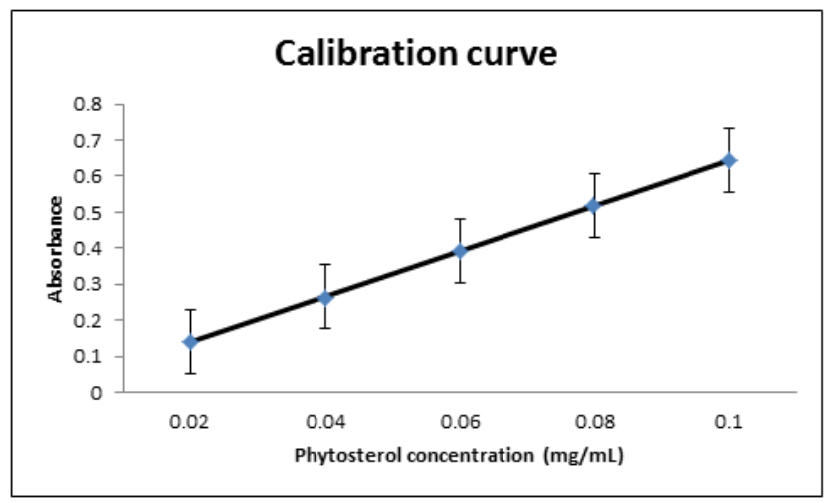

Figure 2 : Calibration curve for beta-sitosterol by using UV-VIS spectrophotometry

Central composite design (CCD) is the most frequent method to be used in response surface methodology for parameter optimization purpose (Walia et. al., 2014). The polynomial regression equation is the most promising method to perform association analysis between each parameter and the desired product (Ghaedi et al., 2015). CCD method usually performs to was used to learn individual and synergetic effect from four parameters reduced to two desired optimum parameter to obtain the high-end yield. CCD model can also reduce the number of the experiment run from each main parameter and their result to gain the end product (Kusuma \&Mahfud, 2016). This study focused on ethanol concentration (A) range between 55 to $95 \%$ of ethanol concentration and duration of time (B) for the extraction process between 4 to $8 \mathrm{~min}$. In Table 3shows the 13 run of the experiment layout and result of 23 Full Factorial Central Composite Design (CCD). Another parameter such as the power of microwave extractor was set at $600 \mathrm{~W}$ and microwave extractor temperature was set at $75^{\circ} \mathrm{C}$ (Ren et al., 2015). The optimal model predictor quadratic equation gave the model conditions for the extraction process in order to obtain the highest yield of phytosteroid as shown in Eqn. 2:

$Y=b_{0}+\sum_{i=1}^{n} b_{i} x_{i}+\sum_{i=1}^{n} b_{i i} x_{i}^{2}+\sum_{i=1}^{n} \sum_{j=i+1}^{n} b_{i j} x_{i} x_{j}$

Where ;

$\mathrm{Y}=$ predicted response (phytosteroid concentration);

$\mathrm{X}=$ independent variables

$\mathrm{b}_{0}=$ model constant

$\mathrm{bi}=$ linear coefficient

bii = quadratic coefficients

bij = interaction coefficients 
The Design Expert Software was used to predict the statistical significance of the equation achieved by using regression analysis for both responses (Ghaedi etal., 2015).

Table 3: ANOVA for Response Surface Quadratic Model (Partial sum of squares) Responses Phytosteroid concentration

\begin{tabular}{|c|c|c|c|c|c|}
\hline Source & $\begin{array}{l}\text { Sum } \\
\text { ofSquares }\end{array}$ & Df & MeanSquare & FValue & p-valueProb $>$ F \\
\hline Model & 0.086 & 5 & 0.017 & 8.95 & $\begin{array}{l}\text { 0.0060 } \\
\text { (significant) }\end{array}$ \\
\hline $\begin{array}{l}\text { A- } \\
\text { Concentration }\end{array}$ & 0.042 & 1 & 0.042 & 21.82 & 0.0023 \\
\hline B-Time & 5.800E-003 & 1 & 5.800E-003 & 3.02 & 0.1260 \\
\hline $\mathrm{AB}$ & 0.017 & 1 & 0.017 & 8.80 & 0.0209 \\
\hline$A^{2}$ & 0.027 & 1 & 0.027 & 14.22 & 0.0070 \\
\hline $\mathrm{B}^{2}$ & 2.801E-004 & 1 & 2.801E-004 & 0.15 & 0.7140 \\
\hline Residual & 0.013 & 7 & 1.923E-003 & & \\
\hline Lack of Fit & 0.011 & 3 & 3.603E-003 & 5.44 & $\begin{array}{l}0.0677 \\
\text { (not significant) }\end{array}$ \\
\hline Pure Error & 2.649E-003 & 4 & 6.623E-004 & & \\
\hline Cor Total & 0.099 & 12 & & & \\
\hline $\mathrm{R}^{2}$ & 0.8647 & & & & \\
\hline Adjusted $\mathrm{R}^{2}$ & 0.7681 & & & & \\
\hline
\end{tabular}

Based on the result in Table 3, the analysis of variance (ANOVA) and regression coefficients of phytosteroid concentration shows that the model was significant where the $\mathrm{p}$-value is 0.0060 that shows the $\mathrm{p}<0.05$. Lack of Fit (LOF) indicated the tolerability of a model that can be fitted with the experimental result. This model lack of fit F-value is 5.44 that suggested only $6.77 \%$ chance of lack of fit F-value to occur and lack of fit for the model is not significant. In order to have a good model, the model must consist of significant p-value but insignificant LOF (Mourabet et al.,2014). The p-value of 0.0060 also shows the model was selecting the best optimum parameter. The F-value of the model is 8.95 that exhibitions the selected parameter (solvent concentration and duration of extraction) is significant but with low probability. Multiple correlation coefficients R2 value of the model is 0.8647 that represent $86.47 \%$ of variability that emphasizes the predicted model were highly explained but only $13.53 \%$ of the predicted model was badly explained. High R2 value showed a high correlation between the predicted value and experimental data (Roosta et al., 2014).

The second order of equation can predict the maximum concentration value of phytosteroid extraction (Lenth, 2009). The equation for the desired model can be generated from the experimental model. The positive coefficient indicated the synergistic effect of the parameter and undesirable effect of the parameter were 
represents by the negative coefficient. The phytosteroid concentration can be calculated and optimize by applying the empirical model of the significant parameter. Eqn. 3 shows the empirical relationship between the significant parameter and the final yield of phytosteroid from the extracted sample in coded form. Figure 3 shows the normal probability plot residual for phytosterol concentration. The plotted graph was shown as a straight line that indicated the model normality assumption was contented with the studied parameter (Senthamarai et al., 2013).

\section{Equation 3 : Empirical model in Terms of Coded Factors}

Phytosterol (Beta-sitosterol) $=+0.25-0.075 * \mathrm{~A}-0.027 * \mathrm{~B}-0.065 * \mathrm{~A} * \mathrm{~B}-0.068 * \mathrm{~A}^{2}+6.318 \mathrm{e}^{-}$ $003 * \mathrm{~B}^{2}$
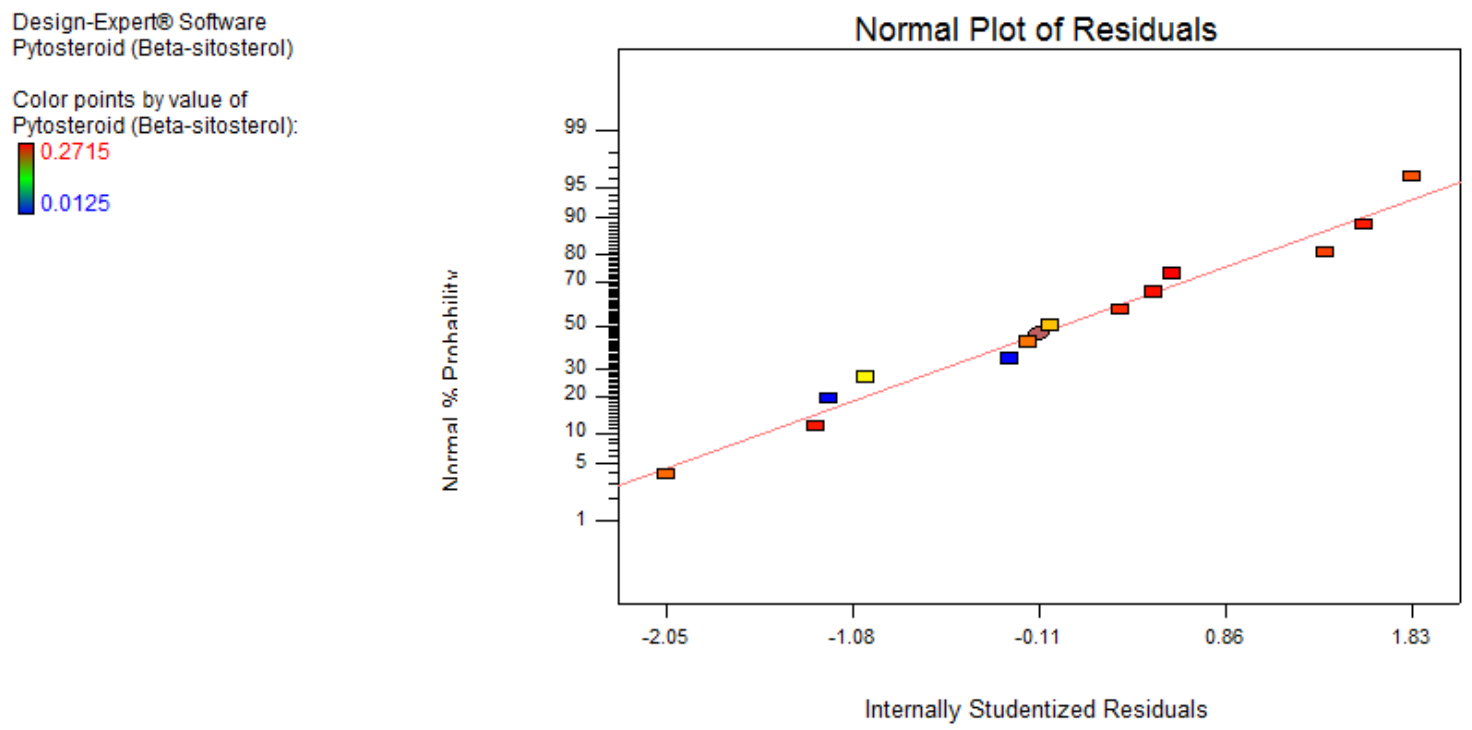

Figure 3: Normal Probability plot residual for Phytosterol concentration

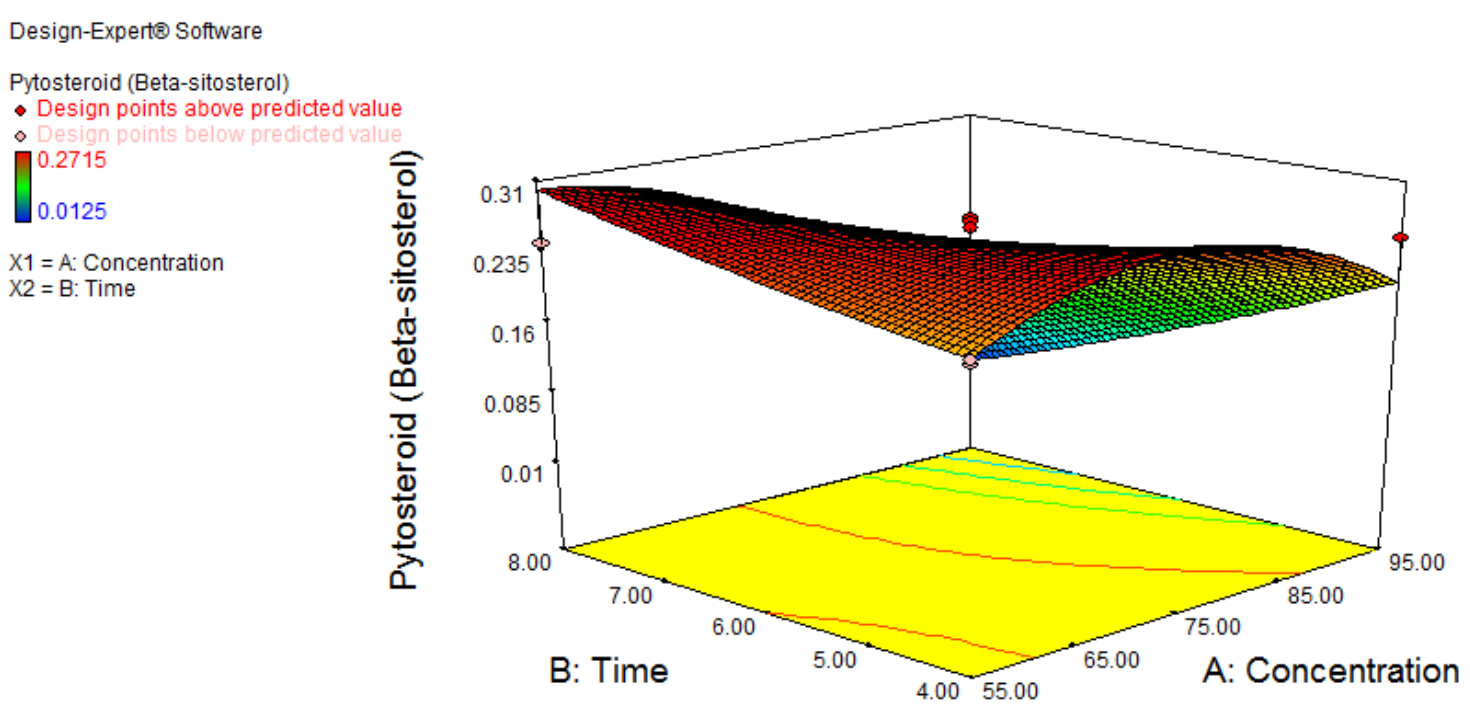


Figure 4 : The contour and 3D surface plot of the phytosteroid concentration

Figure 3 represents the phytosteroid concentration based on the changes in the ethanol concentration and time duration of the extraction process. From the experiment model, it shows that ethanol solvent concentration(F-value=21.82) is the most significant parameter and extraction duration time $(\mathrm{F}$-value $=3.02)$ is the less significant parameter.

\subsection{CONCLUSION}

MAH process can perform the extraction of plant phytosterol such as beta-sitosterol. Central composite design (CCD) was able to study the optimum parameter of the extraction process to extract phytosteroid (beta-sitosterol) in Leucaena leucocephala legume pod by using Design Expert 7.0. Ethanol has been using as the plant extraction solvent because of it safe and effectively act as the extraction solvent to extract the desired product. The CCD model term is significant. This is because the values for Prob $>\mathrm{F}$ is 0.006 which is less than 0.0500 . From the value for lack of fit F-value (5.44) show only $6.77 \%$ chances to occur failure. The model only runs for two parameters which are solvent concentration and time with the F-value of 21.82 and 3.02 significantly.

\section{ACKNOWLEDGEMENT}

Deeply thankful to Universiti Malaysia Pahang for giving the opportunity to perform this research under FKKSA. Also would like to thank to PGRS180336 and RDU1803109 for providing fund for this study.

\section{REFERENCES}

Aderogba, M. A., Mcgaw, L. J., Bezabih, B. T., Abegaz, B. M., (2015). Antioxidant activity and cytotoxicity study of Leucaena Leucocephala (lam.) De wit leaf extract constituents. Nig. J. Nat. Prod. And Med., 13, 65-68.

Araujo, L. B. D. C., Soares, L. A. L., Galvao, M. A. M, Ferreira, M. R. A., Araujo, E. l., Randau, K. P., Soares, L. A. L. (2013). Total phytosterol content in drug materials and exract from roots of Acanthepermim hipidum by UV-VIS spectrophotometry. Revista Brasileira de Farmacognosia, 23(5):736-742.

Begum, J., Das, P. J., (2015). Microwave medited solvent free synthesis of formazans catalysed by simple ionic liquids derived from dialkylammonium salts. RSC Advances, 55, 44604-44609.

Chen, C., Wang, Y. (2010). Steroids from the Whole Plants of Leucaena Leucocephala. American Journal of Analytical Chemistry, 2010(May), 31-33.

Chi Yansheng, Zhang Zhida, Li Changping, Liu Qingshan, Yan Peifang and Urs WelzBiermann, 2011. Microwave-assisted extraction of lactones from Ligusticum chuanxiong Hort. using protic ionic liquids. Green Chem., 13, 666-670.

Elksibi, I., Haddar, W., Ben-Ticha, M., Gharbi, R. Mehnni, M. F. (2014). Development and optimization of non-coventional extraction process of natural dye from olive soid waste using response surface methodology(RSM). Food Chemistry, 161:345352.

Farhata, A., Ginies, C., Romdhane, F., Mehrez, F. C., (2009). Eco-friendly and cleaner 
process for isolation of essential oil using microwave energy: Experimental and theoretical study. Journal of Chromatography A, 5077-5085.

Gomez, L. D., Steele-King, C. G., McQueen-Mason, S. J., 2008, Sustainable liquid biofuels from biomass: the writing's on the walls., New Phytol., 178(3), 473-485.

Harbone, J., B., 1998. Phytochemical Methods: A guide to Modern Techniques of Plant Analysis 3dr Edn, Chapman and Hall, London. pp: 129-138,302.

Kuppusamy, U. R., Arumugam, B., Azaman, N., Chai, J. W. (2014). Leucaena leucocephala fruit aqueous exract stimulate adipogenesis, lipolysis, and glucose uptake in primary rat adipocytes. The scientific World Journal, 1-8.Veggi, P. C., Martinez, J., \&

Kusuma, H. S., Mahfud, M. (2016). Response Surface Methodology for Optimization Studies of Microwave-assisted Extraction of Sandalwood Oil. J. Mater. Environ. Sci., 7(6):1958-1971.

Lenth, R. V., (2009). Response-Surface Method in R, using rsm. Journal Statistical Software, 32(7):1-17.

Li, Hsing-Tan, Ruan, Syun-Wun, Huang, Jin-Cherng, Chen, Hsin-Liang, Chen, ChungYi, (2012). Antioxidant and tyrosinase inhibitor from Leucaena Leucocephala. African Journal of Biotechnology, 11(77), 14182-14185.

Mourabet, M., Abdelhadi, E. R., Boujaady, H. E., Abderrahim, T. (2014). se of response surface methodology for optimization of fluoride adsorption in an aqueous solution by Brushite. Arabian Journal of Chemistry, 217(s2)

Namiesnik, J. and Gorecki, T. (2003). Sample preparation for chromatographic analysis of plant material. J. PlanarChromatogr, 13:404-13.

Palukurty, A., Somalanka, S. R., (2016). Optimization of Nutritional Parameters for Production of Alpha Amylase Using Apergillus oryzae MTCC 3017 bt Central Composite Design. International Journal of Industria Biotechnology and Biomaterials, 2(1), 1-10.

Ren, Y., Chen, Y., Hu, B., Wu, H., Lai, F., Li, X. (2015). Microwave-assisted extraction and new determination method for total steroid saponins from Dioscorea zingiberensis C. H. Wright. Steroids, 104:145-152.

Roosta, M., Ghaedi, M., Daneshfar, A., Asghari, A. (2014). Optimization of Combined transonic assited/tin sulphide nanoparticle loaded on activated carbon removal of erythrosine by response surface methodology. Journal of Industrial and Engineering Chemistry, 21:459-469.

Roosta, M., Ghaedi, M., Daneshfar, A., Sahraei, R. (2015). Ultrasound assisted microextraction-nano material solid phase dispersion for extraction and determination of thymol and carvacrol in pharmaceutical samples: experimental design methodology. J. Chromatogr. B. Analyt. Technol. Biomed. Life Sci., 975:3439.

Senthamarai, C., Senthil, K. P., Priyadharshaini, M., Vijayalakshmi, Vinoth, K. V., Baskaralingam, P., (2013). Adsorption behavior of methylene blue dye onto surface modified Stryhnos potatorum seeds, Environ. Prog. Sustain. Energy, 32:624-632.

Walia, N. K., Sekhon, K. K., Chaudhary, D. P., Cameotra, S. S., Srivastava, P., Kumar, A. (2014). Optimization of Fermentation Parameters for Bioconversion of Corn to Ethanol Using Response Surface Methodology. Journal of Petroleum \& Environmental Biotechnology, 5(3):1000178.

Wang, L. J., and Weller, C. L., (2006). Rcent advances in extraction of nutraceuticals from plant. Trendsin food Science \& Technology, 17(6) 300-312. 